\title{
Dripping down the rivulet
}

\author{
Gaétan Lerisson, ${ }^{*}$ Pier Giuseppe Ledda, Gioele Balestra, and François Gallaire \\ Laboratory of Fluid Mechanics and Instabilities, École Polytechnique Fédérale de Lausanne, \\ Lausanne, CH-1015, Switzerland
}

(Received 1 February 2019; published 24 October 2019)

\begin{abstract}
This paper is associated with a video winner of a 2018 American Physical Society's Division of Fluid Dynamics (DFD) Milton van Dyke Award for work presented at the DFD Gallery of Fluid Motion. The original video is available online at the Gallery of Fluid Motion, https://doi.org/10.1103/APS.DFD.2018.GFM.V0070.
\end{abstract}

DOI: 10.1103/PhysRevFluids.4.100504

A viscous fluid flowing down the underside of a tilted plane substrate develops complex patterns. In this video, we show some of the typical patterns that we isolated in our experiment.

This problem relates to the Rayleigh-Taylor instability [1,2], the destabilization of a horizontal interface between a heavier fluid placed above a lighter fluid. In the present case, an upper wall is close to the interface such that the heavy fluid forms a thin film. The heavy fluid is viscous so we neglect the presence of the light fluid, and inertial effects are kept small. The equilibrium of the interface is then a balance between the destabilizing hydrostatic pressure and stabilizing surface tension effect.

The horizontal case is known to produce highly regular patterns of hexagons or squares of lenses $[3,4]$. In this study, we incline the substrate so that the gravity has, in addition to a wall normal component, a tangential component that breaks the symmetry. The inclination of the substrate can prevent dripping (i.e., large deformation of the interface leading to separation of the fluid) [5]. In our configuration, we continuously feed the thin film with fresh fluid in order to observe the spatial development of the pattern along the film and to reach stationary shapes. From a theoretical perspective, some of the temporal properties of the pattern formation become spatial and stationary.

\section{EXPERIMENTAL SETUP}

The experiment consists of a glass plate of $35 \times 60 \mathrm{~cm}^{2}$ with its large side forming an angle $\theta$ with gravity and its short side being horizontal. A viscous fluid (silicon oil of viscosity $\mu=1 \mathrm{~Pa}$ s) is injected at the top on the underside of the substrate through a thin horizontal slit of width $L_{i}=$ $27 \mathrm{~cm}$. The flow rate per unit length $q$ is homogeneous along the slit and we impose the overall flow rate $Q=q L_{i}$.

The two primary parameters are the angle and the flow rate $(\theta, Q)$. In the presented cases, the flow rate is kept between 0.5 and $2.5 \mathrm{~mL} \mathrm{~s}^{-1}$ and the angle between $20^{\circ}$ and $60^{\circ}$. Note that in all experimental pictures, the flow direction is top-down. In the absence of imposed forcing, the pattern is selected by amplification of ambient noise. However, we can also apply a spatial or temporal

\footnotetext{
*gaetan.lerisson@epfl.ch
}

Published by the American Physical Society under the terms of the Creative Commons Attribution 4.0 International license. Further distribution of this work must maintain attribution to the author(s) and the published article's title, journal citation, and DOI. 

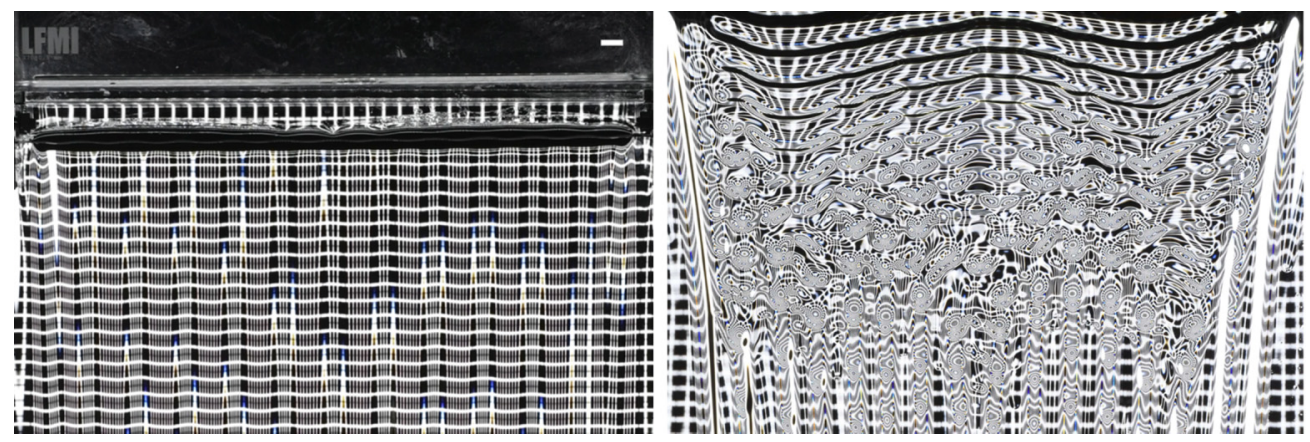

FIG. 1. Visualization by transmission, deformation of a regular grid by the fluid interface. The film is spatially forced (left) with a sinus-shaped blade of specific wavelength, or temporally forced (right) with an oscillating flow rate. Scale-bar: $10 \mathrm{~mm}$.

forcing (or both) at the inlet in order to select a specific pattern. The spatial forcing consists of a blade that touches the interface at the inlet with a chosen wavelength. The temporal forcing consists of a small oscillating variation of the flow rate with a chosen wave period.

\section{TEMPORAL OR SPATIAL FORCING}

The visualization of the interface is first done by transmission. We place a high-contrast regular grid behind the transparent substrate, and observe the deformation of the pattern produced by the perturbation of the interface. Figure 1 (left) shows the case of a spatial forcing with an unstable wavelength for $\theta=20^{\circ}$. The perturbation follows the forcing and develops into streamwise structures, i.e., rivulets.

Figure 1 (right) shows a temporal forcing with $\theta=55^{\circ}$. From the inlet to the outlet, we observe the presence of spanwise structures that deform in a complex pattern, which eventually evolves into streamwise rivulets.

\section{TEMPORAL AND SPATIAL FORCING: RIVULETS, LENSES, AND DRIPPING}

Forcing both spatially and temporally can select much more regular patterns as illustrated in Fig. 2. For an angle of $\theta=60^{\circ}$, the spatial forcing selects regular streamwise structures (rivulets) on which lay temporally forced lenses. Although these lenses have almost a constant velocity, the

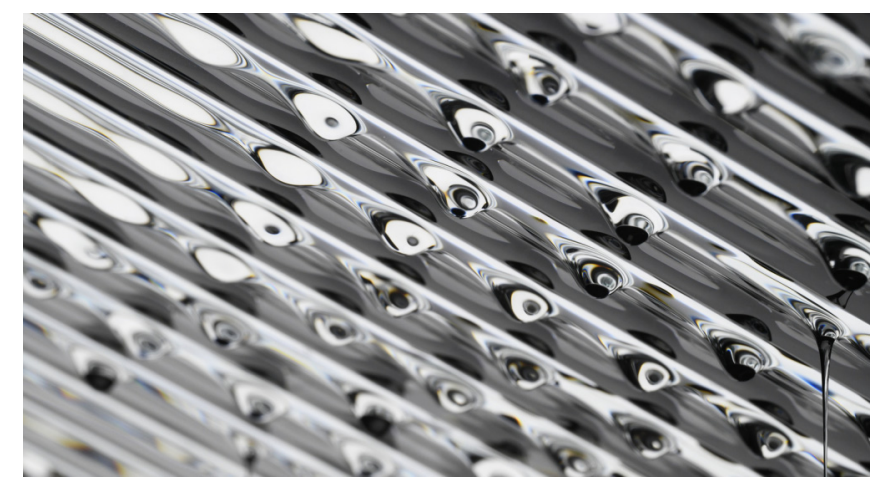

FIG. 2. Rivulets, lenses, and dripping thanks to a spatially and temporally forced film. We directly observe the interface lightened from the side. The typical distance between rivulets is $15 \mathrm{~mm}$. 

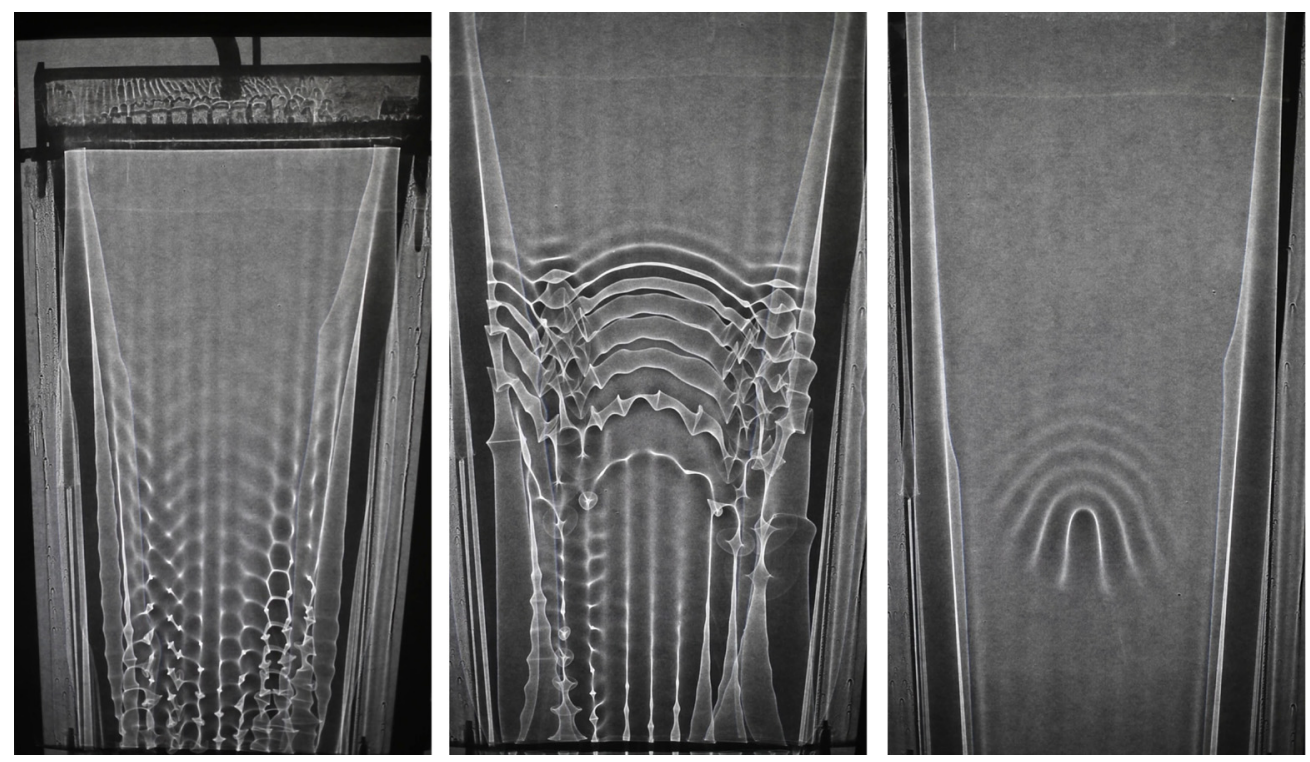

FIG. 3. Shadowgraph of natural noise (left), of the response to a forced impulse on all the inlet (middle), and of the response to a forced localized impulse (right). The picture shows the shadow of the entire plate (length of $60 \mathrm{~cm}$ and width of $33 \mathrm{~cm}$ as the edges are hidden behind the clamps).

merging of two lenses leads to a dramatic dripping as we can observe in the bottom right corner of the picture.

\section{SHADOWGRAPH: IMPULSE RESPONSE}

To perceive extremely small variations of the interface we set a shadowgraph method that consists in a nearly point source of light on one side of the substrate and a white screen on the other side. The ray's deviation by the interface results in variation of the luminosity on the screen. This technique appears to be highly sensitive.

In Fig. 3, we report three pictures of the screen at $\theta=20^{\circ}$. In the left picture, the flow rate is high $\left(Q=2.45 \mathrm{~mL} \mathrm{~s}^{-1}\right)$ and without forcing, complex unsteady patterns appear, resulting in even more complex light formation. In the middle picture, the flow rate is low $\left(Q=1.54 \mathrm{~mL} \mathrm{~s}^{-1}\right)$ and we see the response to a short impulse on the flow rate. Spanwise dominated structures develop and deform in more complex patterns. The right picture shows the response to a spatially localized impulse. We study this selection of streamwise rivulets by looking at the response to a localized perturbation. After some time the localized perturbation is advected downstream. We see the appearance and separation of streamwise (below) and spanwise structures (above).

When the hydrostatic pressure is small enough, i.e., with a more tilted substrate or a smaller flow rate, the film does not drip. In fact, there exists a range of parameters $(\theta, Q)$ for which we do not observe any dripping. Increasing these parameters, we observe a threshold for $(\theta, Q)$ beyond which dripping occurs. Close to this threshold, the route to dripping involves several steps. The film destabilizes and the system selects rivulets, which destabilize as well. Lenses appear on the rivulets, merge, and drip.

\section{ACKNOWLEDGMENT}

We acknowledge the financial support of the Swiss National Science Foundation (Grant No. 200021_178971). 
[1] L. Rayleigh, Investigation of the character of the equilibrium of an incompressible heavy fluid of variable density, Proc. Lond. Math. Soc. s1-14, 170 (1882).

[2] G. I. Taylor, The instability of liquid surfaces when accelerated in a direction perpendicular to their planes. I, Proc. R. Soc. London, Ser. A 201, 192 (1950).

[3] M. Fermigier, L. Limat, J. Wesfreid, P. Boudinet, and C. Quilliet, Two-dimensional patterns in RayleighTaylor instability of a thin layer, J. Fluid Mech. 236, 349 (1992).

[4] L. Limat, P. Jenffer, B. Dagens, E. Touron, M. Fermigier, and J. Wesfreid, Gravitational instabilities of thin liquid layers: Dynamics of pattern selection, Physica D: Nonlinear Phenomena 61, 166 (1992).

[5] P.-T. Brun, A. Damiano, P. Rieu, G. Balestra, and F. Gallaire, Rayleigh-Taylor instability under an inclined plane, Phys. Fluids 27, 084107 (2015). 